\title{
Experimental high-dimensional two-photon entanglement and violations of generalized Bell inequalities
}

\author{
Adetunmise C. Dada ${ }^{1 \star}$, Jonathan Leach ${ }^{2}$, Gerald S. Buller ${ }^{1}$, Miles J. Padgett ${ }^{2}$ and Erika Andersson ${ }^{1}$
}

Quantum entanglement ${ }^{1,2}$ plays a vital role in many quantum-information and communication tasks ${ }^{3}$. Entangled states of higher-dimensional systems are of great interest owing to the extended possibilities they provide. For example, they enable the realization of new types of quantum information scheme that can offer higher-information-density coding and greater resilience to errors than can be achieved with entangled two-dimensional systems (see ref. 4 and references therein). Closing the detection loophole in Bell test experiments is also more experimentally feasible when higher-dimensional entangled systems are used ${ }^{5}$. We have measured previously untested correlations between two photons to experimentally demonstrate high-dimensional entangled states. We obtain violations of Bell-type inequalities generalized to $d$-dimensional systems $s^{6}$ up to $d=12$. Furthermore, the violations are strong enough to indicate genuine 11-dimensional entanglement. Our experiments use photons entangled in orbital angular momentum ${ }^{7}$, generated through spontaneous parametric down-conversion ${ }^{8,9}$, and manipulated using computer-controlled holograms.

Quantum-information tasks requiring high-dimensional bipartite entanglement include teleportation using qudits ${ }^{10,11}$, generalized dense coding (that is, with pairs of entangled $d$-level systems; ref. 12) and some quantum key distribution protocols ${ }^{13}$. More generally, schemes such as quantum secret sharing ${ }^{14}$ and measurement-based quantum computation ${ }^{15}$ apply multiparticle entanglement. These are promising applications, especially in view of recent progress in the development of quantum repeaters (see ref. 16 and references therein). However, practical applications of such protocols are only conceivable when it is possible to experimentally prepare, and moreover detect, high-dimensional entangled states. Therefore, the ability to verify high-dimensional entanglement between physical qudits is of crucial importance. Indeed, much progress has generally been made on the generation and detection of high-dimensional entangled states (please see ref. 17 and references within).

Here we report the experimental investigation of highdimensional, two-photon entangled states. We focus on photon orbital angular momentum (OAM) entangled states generated by spontaneous parametric down-conversion (SPDC), and demonstrate genuine high-dimensional entanglement using violations of generalized Bell-type inequalities ${ }^{6}$. Previously, qutrit Bell-type tests have been carried out using photon OAM to verify threedimensional entanglement (see ref. 18 and references within). In addition to testing whether correlations in nature can be explained by local realist theories ${ }^{19}$, the violation of Bell-type inequalities may be used to demonstrate the presence of entanglement. Bell-type experiments have been carried out using two-dimensional subspaces of the OAM state space of photons ${ }^{20,21}$ and experimentalists have demonstrated two-dimensional entanglement using up to 20 different two-dimensional subspaces ${ }^{22}$. Careful studies have also been carried out to describe how specific detector characteristics bound the dimensionality of the measured OAM states in photons generated by SPDC using Shannon dimensionality ${ }^{23}$.

Our experimental study of high-dimensional entanglement is based on the theoretical work of Collins et al. ${ }^{6}$, which was applied in experiments for qutrits encoded in the OAM states of photons ${ }^{18,24}$. We encode qudits using the OAM states of photons, with eigenstates defined by the azimuthal index $\ell$. These states arise from the solution of the paraxial wave equation in its cylindrical co-ordinate representation, and are the Laguerre-Gaussian modes $L G_{p, \ell}$, so called because they are light beams with a Laguerre-Gaussian amplitude distribution.

In our set-up (Fig. 1), OAM entangled photons are generated through a frequency-degenerate type-I SPDC process, and the OAM state is manipulated with computer-controlled spatial light modulators (SLMs) acting as reconfigurable holograms. Conservation of angular momentum ensures that, if the signal photon is in the mode specified by $|\ell\rangle$, the corresponding idler photon can only be in the mode $|-\ell\rangle$. Assuming that angular momentum is conserved ${ }^{9}$, a pure state of the two-photon field produced will have the form

$$
|\Psi\rangle=\sum_{\ell=-\infty}^{\ell=\infty} c_{\ell}|\ell\rangle_{A} \otimes|-\ell\rangle_{B}
$$

where subscripts $A$ and $B$ label the signal and idler photons respectively, $\left|c_{\ell}\right|^{2}$ is the probability to create a photon pair with $\mathrm{OAM} \pm \ell \hbar$ and $|\ell\rangle$ is the OAM eigenmode with mode number $\ell$.

It has been shown ${ }^{6}$ that, for correlations that can be described by theories based on local realism ${ }^{1}$, a family of Bell-type parameters $S_{d}$ satisfies the inequalities

$$
S_{d}^{(\text {local realism) }} \leq 2, \text { for all } d \geq 2
$$

Alternatively, if quantum mechanics is assumed to hold, then the violation of an inequality of type (1) indicates the presence of entanglement. $S_{d}$ can be expressed as the expectation value of a quantum mechanical observable, which we denote as $\hat{S}_{d}$. The expressions for $S_{d}, \hat{S}_{d}$ and the operators $\hat{S}_{2}$ and $\hat{S}_{3}$ are provided in Supplementary Section SI. 


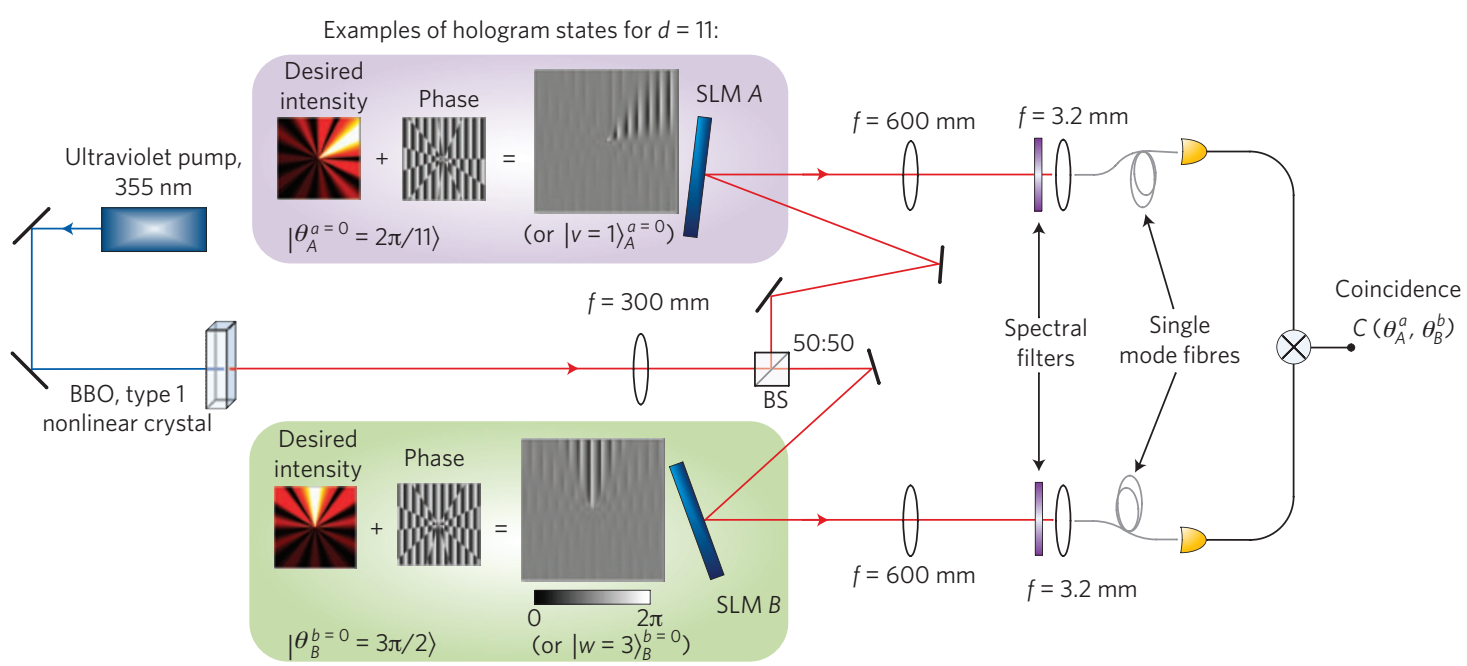

Figure 1 | Schematic representation of experimental set-up for violations of Bell-type inequalities. $C\left(A_{a}=v, B_{b}=w\right)$ or $C\left(\theta_{A}^{a}, \theta_{B}^{b}\right)$ is the coincidence count rate when SLM $A$ is in state $|v\rangle_{a}^{A}$ or $\left|\theta_{A}^{a}\right\rangle$ and $\operatorname{SLM} B$ is in state $|w\rangle_{b}^{B}$ or $\left|\theta_{B}^{b}\right\rangle$ respectively.

The parameters $S_{d}$ are calculated using coincidence probabilities for measurements made locally by two observers, Alice and Bob, on their respective subsystems, which in our case are the signal and idler photons from the SPDC source. Alice's detector has two settings labelled by $a \in\{0,1\}$ with $d$ outcomes for each setting, and similarly for Bob's detector with settings $b \in\{0,1\}$. The measurement bases corresponding to the detector settings of Alice and Bob are defined as

$$
\begin{aligned}
|v\rangle_{a}^{A} & =\frac{1}{\sqrt{d}} \sum_{j=0}^{d-1} \exp \left[i \frac{2 \pi}{d} j\left(v+\alpha_{a}\right)\right]|j\rangle \\
|w\rangle_{b}^{B} & =\frac{1}{\sqrt{d}} \sum_{j=0}^{d-1} \exp \left[i \frac{2 \pi}{d} j\left(-w+\beta_{b}\right)\right]|j\rangle
\end{aligned}
$$

where $v$ and $w$ both run from 0 to $d-1$ and denote the outcomes of Alice's and Bob's measurements respectively, and the parameters $\alpha_{0}=0, \alpha_{1}=1 / 2, \beta_{0}=1 / 4$ and $\beta_{1}=-1 / 4$.

The measurement bases $\left\{|v\rangle_{a}^{A}\right\}$ and $\left\{|w\rangle_{a}^{A}\right\}$ have been shown ${ }^{5,25}$ to maximize the violations of inequality (1) for the maximally entangled state of two $d$-dimensional systems given by $|\psi\rangle=$ $(1 / \sqrt{d}) \sum_{j=0}^{d-1}|j\rangle_{A} \otimes|j\rangle_{B}$. It turns out that we are able to parameterize these $d$-dimensional measurement basis states with 'mode analyser' angles $\theta_{A}$ and $\theta_{B}$, and write them in the form

$$
\begin{gathered}
|v\rangle_{a}^{A} \equiv\left|\theta_{A}^{a}\right\rangle=\frac{1}{\sqrt{d}} \sum_{\ell=-[d / 2]}^{\ell=+[d / 2]} \exp \left[i \theta_{A}^{a} g(\ell)\right]|\ell\rangle, \text { and } \\
|w\rangle_{b}^{B} \equiv\left|\theta_{B}^{b}\right\rangle=\frac{1}{\sqrt{d}} \sum_{\ell=-\left[\frac{d}{2}\right]}^{\ell=+\left[\frac{d}{2}\right]} \exp \left[i \theta_{B}^{b} g(\ell)\right]|\ell\rangle
\end{gathered}
$$

where

$$
\begin{gathered}
\theta_{A}^{a}=(v+a / 2) 2 \pi / d \\
\theta_{B}^{b}=\left[-w+1 / 4(-1)^{b}\right] 2 \pi / d
\end{gathered}
$$

The function $g(\ell)$ is defined as

$$
g(\ell)=\ell+\left[\frac{d}{2}\right]+(d \bmod 2) u(\ell)
$$

where $[x]$ is the integer part of $x$, and $\mathrm{u}(\ell)$ is the discrete unit step function.
Figure 2 shows an example of the experimental data points for the self-normalized coincidence rates as function of the relative angle $\left(\theta_{A}-\theta_{B}\right)$ using $d=11$ (see also Supplementary Fig. S1). For a maximally entangled state

$$
|\Phi\rangle=\frac{1}{\sqrt{d}} \sum_{\ell=-[d / 2]}^{[d / 2]} h(\ell)|\ell\rangle_{A} \otimes|-\ell\rangle_{B}
$$

where $h(\ell)=1$ for all $\ell$ when $d$ is odd, and $h(\ell \neq 0)=1, h(0)=1$ when $d$ is even, the coincidence rate of detecting one photon in state $\left|\theta_{A}\right\rangle$ and the other in state $\left|\theta_{B}\right\rangle$ is proportional to

$$
C\left(\theta_{A}, \theta_{B}\right)=\mid\left\langle\theta_{A}\left|\left\langle\theta_{B}|| \Phi\right\rangle\right|^{2} \propto \frac{\cos \left(d\left(\theta_{A}-\theta_{B}\right)\right)-1}{d^{3}\left[\cos \left(\theta_{A}-\theta_{B}\right)-1\right]}\right.
$$

The key result of our paper is shown in Fig. 3, which shows a plot of experimental values of parameter $S_{d}$ as a function of the number of dimensions $d$. The plot compares theoretically predicted violations for a maximally entangled state, the experimental readings and the local hidden variable (LHV) limit. The maximum possible violations (shown in Supplementary Table S1) are slightly larger than the corresponding violations produced by a maximally entangled state. Violations persist up to as much as $d=12$ when entanglement concentration ${ }^{26}$ is applied. We find $S_{11}=2.39 \pm 0.07$

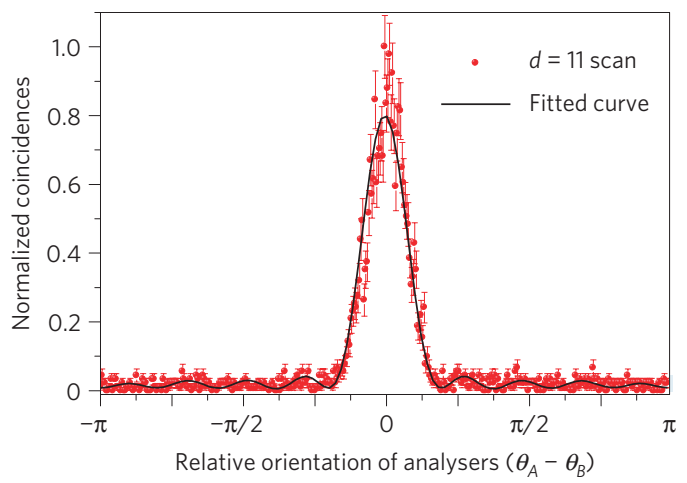

Figure 2 | Coincidence count rate (self-normalized) as a function of the relative orientation angle between state analysers $\left(\theta_{A}-\theta_{B}\right)$. Equation (5) for a state with maximal 11-dimensional entanglement is fitted to the experimental data with the vertical offset and amplitude left as free parameters. Errors were estimated assuming Poisson statistics. 


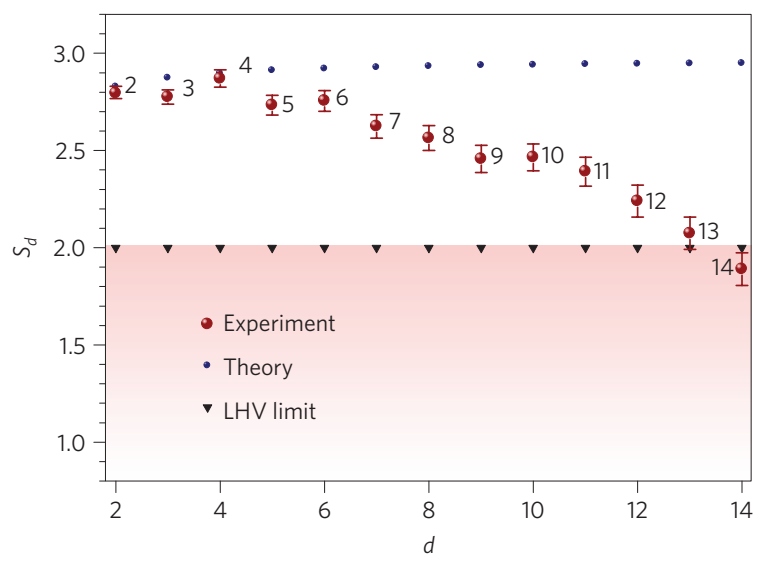

Figure 3 | Experimental Bell-type parameter $S_{d}$ versus number of dimensions $d$. $S_{d}>2$ violates local realism for any $d \geq 2$. The plot compares the theoretically predicted violations by a maximally entangled state and the LHV limit with the experiments. Violations are observed for up to $d=12$. Errors were estimated assuming Poisson statistics.

and $S_{12}=2.24 \pm 0.08$, which clearly violate $S_{d} \leq 2$ (see also Supplementary Table S4). In the corresponding experiment using $L G_{p, \ell}$ modes with only $p=0$, violations are obtained up to $d=11$. Without entanglement concentration, we observe violations only up to $d=9$ (please see Supplementary Fig. S2 in Section SIV). Above $d \sim 11$, the strength of the signal becomes so low that noise begins to overshadow the quantum correlations. In Fig. 2, the theoretical prediction in equation (5) for a state with maximal 11-dimensional entanglement is fitted to the experimental coincidence data obtained using the mode analyser settings defined in equation (4) for $d=11$, with only the vertical offset and amplitude left as free parameters. The observed fringes are seen to closely match those theoretically obtained for a state with maximal 11-dimensional entanglement.

The violation of a Bell inequality in $d \times d$ dimensions directly indicates that the measured state was entangled. It remains to determine how many dimensions were involved in the entanglement. Measuring the coincidence probabilities, that is, of there being the joint state $\left|\ell_{\mathrm{s}}\right\rangle \otimes\left|\ell_{\mathrm{i}}\right\rangle$ (Fig. 4), together with the parameters $S_{d}$ for different $d$, can be seen as a partial tomography of the SPDC source state. Numerical investigations indicate that a state with the experimentally observed coincidence probabilities and parameters $S_{2}, S_{3}, \ldots, S_{11}$ must contain genuine 11-dimensional entanglement. In other words, it is not possible to obtain the observed levels of violation with a state that contains entanglement involving only 10 dimensions or less. Our analysis assumes a special form of the states, based on the coincidence measurement results shown in Fig. 4. Further details are given in Supplementary Section SII.

Our results hold much promise for applications requiring entangled qudits in general. As mentioned earlier, progress in the development of quantum repeaters (see ref. 16 and references therein) would make quantum key distribution using high-dimensional entangled states ${ }^{13}$ a possible application. Conventional quantum communication will fail for sufficiently large transmission distances because of loss, and quantum repeaters are one possible solution to this problem. Although experimental quantum key distribution has been demonstrated with OAM qutrits ${ }^{24}$, our findings provide experimental evidence that such protocols could be implemented using photons entangled in OAM in up to 11 dimensions, resulting in a considerable increase in information coding density.

A possible extension to our work would be to investigate the generation of multiphoton, high-dimensional OAM entanglement. We can conceive of achieving this using a cascade of downconversion crystals for generating multipartite entangled photons,

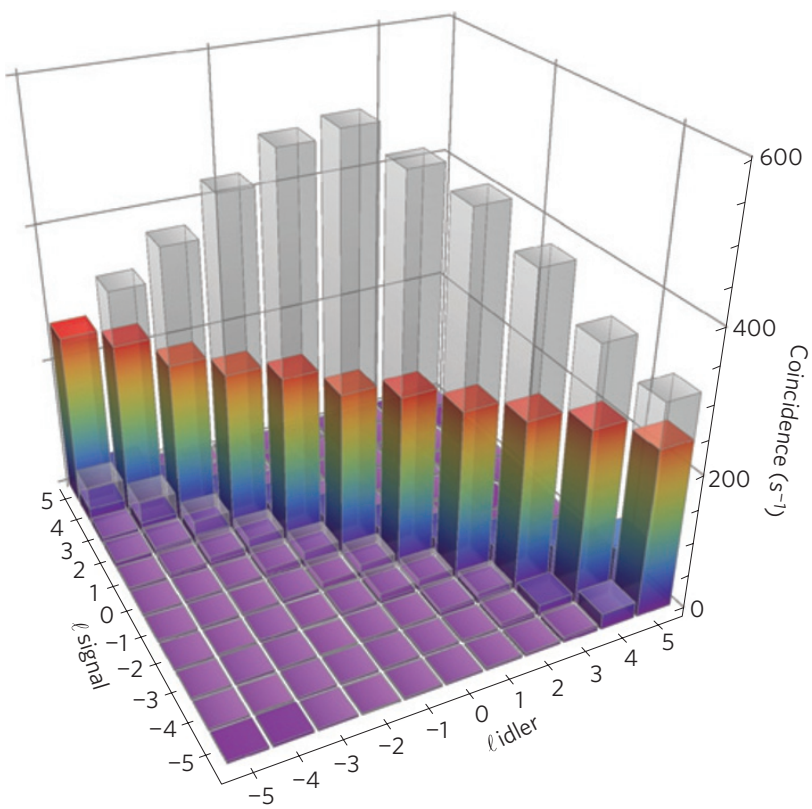

Figure 4 | Experimental coincidence rates proportional to the probability of measuring the state $\left|\ell_{s}\right\rangle \otimes\left|\ell_{i}\right\rangle$ with $\ell_{s}, \ell_{i}=-5, \ldots,+5$. The coloured and greyed-out bars depict the measurement results with and without the application of Procrustean filtering respectively. The measurement time was $20 \mathrm{~s}$ for each combination of $\ell_{\mathrm{s}}$ and $\ell_{\mathrm{i}}$.

which has been done for polarization entangled photons ${ }^{17}$. It also seems to be within reach to combine the high-dimensional photon OAM entanglement with entanglement in the polarization and path degrees of freedom, creating even larger hyper-entangled states (see ref. 17 and references within).

On a more fundamental note, Bell test experiments carried out so far have one or both of two main loopholes, namely the locality and detection loopholes. However, a recent theoretical work reveals that even low-dimensional qudits can provide a significant advantage over qubits for closing the detection loophole ${ }^{5}$. In fact, it was found that as much as $38.2 \%$ loss can be tolerated using four-dimensional entanglement. Our results raise interesting possibilities regarding the role higher-dimensional entangled qudits could play in closing this loophole. We emphasize that neither the detection nor the locality loophole has been closed in our experiments, because the overall efficiency of our experimental set-up is $1-2 \%$, and the switching time for our measurement devices (SLMs) is of the order of tens of milliseconds. However, closing these loopholes was not the immediate goal of our experiments. We are instead using the violation of Bell inequalities, up to fair sampling assumptions, as a means of verifying the presence of high-dimensional entanglement, within the framework of quantum mechanics.

In summary, we have been able to experimentally demonstrate violations of Bell-type inequalities generalized to $d$-dimensional systems $^{6}$ with up to $d=12$, enough to indicate genuine 11dimensional entanglement in the OAM of signal and idler photons in parametric down-conversion. It seems that this could be extended to even higher dimensions by using a brighter source of entangled photons.

\section{Methods}

In our experiments, we use computer-controlled SLMs (Hamamatsu) operating in reflection mode with a resolution of $600 \times 600$ pixels. In the detection, the SLMs are prepared in the states defined in equation (4) respectively. An SLM prepared in a given state transforms a photon in that state to the Gaussian $|\ell=0\rangle$ mode. The reflected photon is then coupled into a single-mode fibre which feeds a single photon detector. As only the $|\ell=0\rangle$ mode couples into the fibre, a count in the detector indicates a detection of the state in which the SLM was 
prepared. The hologram generation algorithm introduced in ref. 27 is applied to configure the SLMs.

Figure 1 shows a schematic diagram of the experimental set-up as well as examples of SLM settings used where $d=11$. For the SPDC, we use a pump beam, with $\ell=0$, produced by a frequency-tripled, mode-locked Nd-YAG laser with an average output power of $150 \mathrm{~mW}$ at $355 \mathrm{~nm}$. The collimated laser beam is normally incident on a 3-mm-long $\beta$-barium borate (BBO) crystal cut for type-I collinear phase matching. A 50:50 beam splitter (BS) then separates the co-propagating OAM entangled photons probabilistically into the signal and idler paths. Spectral filters with $10 \mathrm{~nm}$ bandwidth are used to reduce the detection of noise photons. The coincidence resolving time is $10 \mathrm{~ns}$ and an integration time of $20 \mathrm{~s}$ is used for the measurements.

For tests within a $d$-dimensional subspace and for odd $d$, we choose the modes $\ell=-(d-1) / 2, \ldots, 0, \ldots,(d-1) / 2$ as the computational basis states $|j\rangle$ in equations (2) and (3), where $j=0, \ldots, d-1$. For even $d$, we use $\ell=-d / 2, \ldots,-1,1, \ldots, d / 2$, omitting the $\ell=0$ mode. A projection of the SPDC output state onto a $d$-dimensional subspace results in a non-maximally entangled state owing to the limited spiral bandwidth ${ }^{28}$. To enhance the entanglement, we use the so-called Procrustean method of entanglement concentration ${ }^{26}$. This is generally done by means of a filtering technique that equalizes the mode amplitudes, thereby probabilistically enhancing the entanglement of the two-photon state ${ }^{29}$. This can be achieved by applying local operations to one or both of the signal and idler photons. We choose local operations matched to the spiral bandwidth measurement for our SPDC source (please see Supplementary Section SIV), so as to obtain a close approximation to a maximally entangled state. The method applied in ref. 30 uses lenses for equalizing amplitudes in a superposition of three OAM modes. We however use alterations of the diffraction efficiencies of blazed phase gratings in the SLMs to achieve this goal for up to 14 modes. Figure 4 contrasts the results of coincidence measurements with and without Procrustean filtering, with the SLMs in the state $\left\{\left|\ell_{A}\right\rangle \otimes\left|\ell_{B}\right\rangle\right\}$ where $\ell_{A}, \ell_{B} \in\{-5, \ldots,+5\}$. The disadvantage of the Procrustean method is the associated reduction in the number of detected photons (see Supplementary Section SIII for further details).

\section{Received 15 October 2010; accepted 7 April 2011; published online 8 May 2011}

\section{References}

1. Einstein, A., Podolsky, B. \& Rosen, N. Can quantum-mechanical description of physical reality be considered complete? Phys. Rev. 47, 777-780 (1935).

2. Schrödinger, E. Die gegenwärtige Situation in der Quantenmechanik. Naturwissenschaften 23 807-812; 823-828; 844-849 (1935).

3. Nielsen, M. A. \& Chuang, I. L. Quantum Computation and Quantum Information (Cambridge Univ. Press, 2000).

4. Durt, T., Kaszlikowski, D., Chen, J-L. \& Kwek, L. C. Security of quantum key distributions with entangled qudits. Phys. Rev. A 69, 032313 (2004).

5. Vértesi, T., Pironio, S. \& Brunner, N. Closing the detection loophole in Bell experiments using qudits. Phys. Rev. Lett. 104, 060401 (2010).

6. Collins, D., Gisin, N-l., Linden, N., Massar, S. \& Popescu, S. Bell inequalities for arbitrarily high dimensional systems. Phys. Rev. Lett. 88, 040404 (2002).

7. Allen, L., Beijersbergen, M. W., Spreeuw, R. J. C. \& Woerdman, J. P. Orbital angular momentum of light and the transformation of Laguerre-Gaussian laser modes. Phys. Rev. A 45, 8185-8189 (1992).

8. Kwiat, P. G. et al. New high-intensity source of polarization-entangled photon pairs. Phys. Rev. Lett. 75, 4337-4341 (1995).

9. Walborn, S. P., de Oliveira, A. N., Thebaldi, R. S. \& Monken, C. H. Entanglement and conservation of orbital angular momentum in spontaneous parametric down-conversion. Phys. Rev. A 69, 023811 (2004).

10. Bennett, C. H. et al. Teleporting an unknown quantum state via dual classical and Einstein-Podolsky-Rosen channels. Phys. Rev. Lett. 70, 1895-1899 (1993).

11. You-Bang, Z., Qun-Yong, Z., Yu-Wu, W. \& Peng-Cheng, M. Schemes for teleportation of an unknown single-qubit quantum state by using an arbitrary high-dimensional entangled state. Chinese Phys. Lett. 27, 10307-10310 (2010).
12. Bennett, C. H. \& Wiesner, S. J. Communication via one- and two-particle operators on Einstein-Podolsky-Rosen states. Phys. Rev. Lett. 69 2881-2884 (1992).

13. Ekert, A. K. Quantum cryptography based on Bell's theorem. Phys. Rev. Lett 67, 661-663 (1991).

14. Hillery, M., Bužek, V. \& Berthiaume, A. Quantum secret sharing. Phys. Rev. A 59, 1829-1834 (1999).

15. Raussendorf, R., Browne, D. E. \& Briegel, H. J. Measurement-based quantum computation on cluster states. Phys. Rev. A 68, 022312 (2003).

16. Yuan, Z-S. et al. Experimental demonstration of a BDCZ quantum repeater node. Nature 454, 1098-1101 (2008).

17. Gao, W-B. et al. Experimental demonstration of a hyper-entangled ten-qubit Schrödinger cat state. Nature Phys. 6, 331-335 (2010).

18. Vaziri, A., Weihs, G. \& Zeilinger, A. Experimental two-photon, three-dimensional entanglement for quantum communication. Phys. Rev. Lett. 89, 240401 (2002).

19. Aspect, A., Grangier, P. \& Roger, G. Experimental realization of Einstein-Podolsky-Rosen-Bohm gedankenexperiment: A new violation of Bell's inequalities. Phys. Rev. Lett. 49, 91-94 (1982).

20. Leach, J. et al. Violation of a Bell inequality in two-dimensional orbital angular momentum state-spaces. Opt. Exp. 17, 8287-8293 (2009).

21. Jack, B. et al. Entanglement of arbitrary superpositions of modes within two-dimensional orbital angular momentum state spaces. Phys. Rev. A 81, 043844 (2010).

22. Jack, B. et al. Precise quantum tomography of photon pairs with entangled orbital angular momentum. New J. Phys. 11, 103024 (2009).

23. Pors, J. B. et al. Shannon dimensionality of quantum channels and its application to photon entanglement. Phys. Rev. Lett. 101, 120502 (2008)

24. Gröblacher, S., Jennewein, T., Vaziri, A., Weihs, G. \& Zeilinger, A. Experimental quantum cryptography with qutrits. New J. Phys. 8, 75 (2006).

25. Durt, T., Kaszlikowski, D. \& Zukowski, M. Violations of local realism with quantum systems described by $N$-dimensional Hilbert spaces up to $N=16$. Phys. Rev. A 64, 024101 (2001).

26. Bennett, C. H., Bernstein, H. J., Popescu, S. \& Schumacher, B. Concentrating partial entanglement by local operations. Phys. Rev. A 53, 2046-2052 (1996).

27. Leach, J., Dennis, M. R., Courtial, J. \& Padgett, M. J. Vortex knots in light. New J. Phys. 7, 55 (2005).

28. Torres, J. P., Alexandrescu, A. \& Torner, L. Quantum spiral bandwidth of entangled two-photon states. Phys. Rev. A 68, 050301 (2003).

29. Law, C. K. \& Eberly, J. H. Analysis and interpretation of high transverse entanglement in optical parametric down conversion. Phys. Rev. Lett. 92, 127903 (2004).

30. Vaziri, A., Pan, J-W., Jennewein, T., Weihs, G. \& Zeilinger, A. Concentration of higher dimensional entanglement: Qutrits of photon orbital angular momentum. Phys. Rev. Lett. 91, 227902 (2003).

\section{Acknowledgements}

We acknowledge suggestions from S. M. Barnett. This work was funded by the Engineering and Physical Sciences Research Council (EPSRC). A.C.D. acknowledges funding support from the Scottish Universities Physics Alliance (SUPA).

\section{Author contributions}

A.C.D. and E.A. devised the concept of the experiment. E.A. supervised the theoretical aspects of the project. G.S.B. and M.J.P. advised on aspects of experimental design. A.C.D. and J.L. carried out the experiment. A.C.D. and E.A. wrote the paper with contributions from all authors.

\section{Additional information}

The authors declare no competing financial interests. Supplementary information accompanies this paper on www.nature.com/naturephysics. Reprints and permissions information is available online at http://www.nature.com/reprints. Correspondence and requests for materials should be addressed to A.C.D. 